\title{
NOUVELLE
}

\section{Peut-on séparer GVH et GVT après greffe de cellules souches hématopoïétiques allogéniques?}

Mohamad Mohty, Daniel Olive des lymphocytes T CD4 $4^{+}$allogéniques). L'analyse se fondait sur la clonalité du récepteur de l'antigène des lymphocytes T (TCR, T cell receptor) par la technique du complementarity-determining region 3 (CDR3) pour les différents locus du TCR $\beta$ [6]. Malgré les nombreuses limites de ce modèle, ces données laissent supposer l'existence de cibles antigéniques spécifiques des cellules tumorales, que ne partagent pas les cellules saines cibles de la GVH. Cependant, ces antigènes ne sont pas encore clairement identifiés: plusieurs candidats existent comme les antigènes tumoraux, mais aussi les antigènes mineurs d'histocompatibilité, laissant encore le débat ouvert quant à la possibilité d'une séparation GVH-GVT. L'allo-CSH connaît actuellement un essor considérable (855 patients recensés en France en 2001), non seulement dans le traitement des hémopathies malignes, mais aussi dans certaines tumeurs solides [7]. Cela est principalement lié au développement de conditionnements dits d'intensité réduite. Dans ces conditions de myélo-ablation courte, ces alloCSH permettent une prise de greffe rapide avec une toxicité limitée, et leur objectif ultime est de favoriser l'émergence de l'effet GVT par rapport aux autres effets toxiques comme ceux qui sont associés à la GVHD. Plusieurs études pilotes ont démontré la faisabilité, la tolérance et, dans certains cas, l'efficacité de ce type de conditionnement. Ainsi, la mortalité immédiate ( $<3$ mois) en rapport avec l'allo-CSH est significativement plus faible après un conditionnement d'intensité réduite qu'après un conditionnement classique myélo-ablatif. Cependant, malgré la diminution de la toxicité immédiate, la forte immunosuppression à laquelle sont soumis ces 
malades (sérum anti-lymphocytaire, fludarabine, ciclosporine, mycophénolate mofétil, etc.), peut inhiber une réponse immune efficace, et met donc l'accent sur la nécessité d'adjoindre à l'allo-CSH d'autres stratégies d'immunothérapie comme la vaccination par cellules dendritiques ou la manipulation des effecteurs avant réinjection des lymphocytes du donneur, capables d'induire à long terme un véritable effet GVT avec contrôle durable et efficace de la maladie. $\diamond$

Segregating effectors for graft versus host and graft versus tumor reactions

\section{RÉFÉRENCES}

1. Horowitz MM, Gale RP, Sondel PM, et al. Graftversus-leukemia reactions after bone marrow transplantation. Blood 1990 75: 555-62.

2. Weiden PL, Sullivan KM, Flournoy N, Storb R, Thomas $\varepsilon D$. Antileukemic effect of chronic graft-versus-host disease: contribution to improved survival after allogeneic marrow transplantation. $N$ Engl Med 1981; 304: 1529-33.

3. Maraninchi D, Gluckman $\varepsilon$, Blaise D, et al. Impact of Tcell depletion on outcome of allogeneic bone-marrow transplantation for standard-risk leukaemias. Lancet 1987; 2: 175-8.

4. Kolb HJ, Schattenberg A, Goldman JM, et al. Graftversus-leukemia effect of donor lymphocyte transfusions in marrow grafted patients. European group for blood and marrow transplantation working party chronic leukemia. Blood 1995; 86: 2041-50.

5. Falkenburg, JH, Wafelman $A R$, Joosten $P$, et al. Complete remission of accelerated phase chronic myeloid leukemia by treatment with leukemia- reactive cytotoxic $T$ lymphocytes. Blood 1999; 94: 1201-8.

6. Michalek, J, Collins RH, Durrani HP, et al. Definitive separation of graft-versusleukemia- and graft-versushost- specific $C D 4^{+} T$ cells by virtue of their receptor beta loci sequences. Proc Natl Acad Sci USA 2003; 100: 1180-4.

7. Childs, R, Chernoff A, Contentin N, et al. Regression of metastatic renal-cell carcinoma after nonmyeloablative allogeneic peripheral-blood stem-cell transplantation. N Engl J Med 2000; 343: 750-8.
NOUVELL

\section{Rôles physiologiques de I'AMP-activated protein kinase (AMPK)}

Fabrizio Andreelli, Benoît Viollet, Sophie Vaulont
> L'AMP-activated protein kinase (AMPK), enzyme ubiquitaire, est proposée comme le détecteur métabolique de la cellule lui permettant de s'adapter aux modifications de son environnement [1]. L'AMPK est un complexe trimérique constitué d'une sousunité catalytique $\alpha$ et de deux sous-unités régulatrices $\beta$ et $\gamma$. II existe deux isoformes $\alpha(\alpha 1$ et $\alpha 2)$, deux isoformes $\beta$ ( $\beta$ l et $\beta 2)$ et trois isoformes $\gamma(\gamma 1, \gamma 2, \gamma 3)$ [1]. Chacune de ces isoformes est codée par un gène différent. L'AMPK est activée physiologiquement par $(\rightarrow) \mathrm{m} / \mathrm{s}$ $1999, n^{\circ} 11$, p. 1276 métabolisable de I'AMP capable d'activer I'AMPK, les rôles de l'AMPK ont pu être peu à peu précisés in vitro [2] et in vivo [3, 4]. Dans le muscle, l'activation de l'AMPK stimule l'augmentation du captage insulinoindépendant du glucose [2] et l'oxydation des acides gras [2]. On connaît le rôle de la leptine dans l'augmentation de l'oxydation musculaire des acides gras, mais cet effet nécessite l'activation de l'AMPK $(\rightarrow)$ [5]. Dans le foie, l'activation de l'AMPK par l'AICAR diminue la néoglucogenèse in vitro [6] et la production hépatique de glucose l'augmentation de la concentration intracellulaire en AMP lors des situations de carence énergétique cellulaire (hypoxie, hypoglycémie et exercice physique) [2]. L'activation de I'AMPK réduit les voies métaboliques consommatrices d'énergie (comme la lipogenèse ou la synthèse stéroïdienne) et augmente les voies productrices d'ATP (comme l'oxydation des acides gras). Grâce au 5-amino-imidazolecarboxamide riboside (AICAR), analogue non
Département de génétique

développement et pathologie

moléculaire, Inserm-Cnrs,

Institut Cochin,

Faculté de Médecine

Cochin-Port-Royal,

24, rue du Faubourg

Saint-Jacques, 75014 Paris,

France.

andreelli@cochin.inserm.fr au cours du diabète de type 2 et de l'obésité [2].

Pour mieux connaître les rôles physiologiques de l'isoforme catalytique $\alpha 2$ de I'AMPK sans avoir à utiliser de moyens pharmacologiques comme I'AICAR, nous avons créé au laboratoire un modèle de rongeurs dont le gène codant pour cette sous-unité a été invalidé [7].

Les souris AMPK $\alpha 2^{-/-}$n'ont pas d'anomalies apparentes. Ces souris ont, après un jeûne court, une glycémie et une insulinémie similaires à celles des souris témoins. Lorsque l'apport nutritif n'est pas limité, et lors d'une hyperglycémie provoquée par voie intrapéritonéale, on observe chez les souris mutantes une hyperglycémie associée à une sécrétion réduite d'insuline. De manière surprenante, la sécrétion d'insuline chez ces animaux, mesurée après exposition in vitro d'îlots pancréatiques isolés à une large gamme de concentrations de glucose (de 3 à $25 \mathrm{mM}$ ), est normale. Par ailleurs, le contenu en insuline et l'expres- 